BMJ Paediatrics Open

\section{A protocol for quality improvement programme to reduce central line- associated bloodstream infections in NICU of low and middle income country}

To cite: Hussain ASshabbir, Ali SR, Ariff S, et al. A protocol for quality improvement programme to reduce central line-associated bloodstream infections in NICU of low and middle income country. BMJ Paediatrics Open 2017;1:e000008. doi:10.1136/ bmjpo-2017-000008

- Additional material is published online only. To view please visit the journal online (http://dx.doi.org/10.1136/ bmjpo-2017-000008).

Received 15 March 2017 Revised 29 August 2017 Accepted 19 September 2017

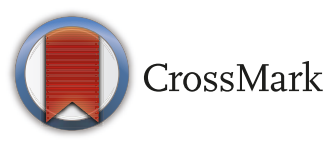

${ }^{1}$ Department of Pediatrics and Child Health, The Aga Khan University, Karachi, Pakistan ${ }^{2}$ Department of Pediatrics, The Aga Khan University Hospital, Karachi, Pakistan

Correspondence to Dr Ali Shabbir shabbir Hussain; ali.hussain@aku.edu

\section{ABSTRACT}

Introduction Central line-associated bloodstream infections (CLABSI) are the most important cause of morbidity and mortality in critically ill patients. Evidencebased interventions when used in form of a bundle have proven to decrease CLABSI. Our unit has a high CLABSI rate (9/1000 central line days). Therefore, we intend to introduce evidence-based CLABSI prevention package in our practice to improve CLABSI rates in our NICU within limited resources.

Methods and analysis The study will be conducted using preanalysis and postanalysis design from January 2016 to December 2017. It is going to be conducted in three phases with phase I being the preimplimentation phase where retrospective data will be collected. Phase II, implementation phase, where the CLABSI prevention package will be introduced and phase III will be follow-up to see the impact. Primary outcome will be reduction in CLABSI rates.

Analysis plan and reporting For all three phases, descriptive analysis will be performed. Nominal data will be presented as mean $\pm S D$, whereas categorical data will be presented as frequencies and percentages. To compare the effect of intervention we will use independent sample $\mathrm{t}$-test for continuous outcomes, whereas $\mathrm{X}^{2}$ test will be used for categorical outcomes. Relative risk ratios, $95 \% \mathrm{Cl}$, and $p$ values will be determined. Incidence density will be calculated and Poisson regression will be used to determine factors associated with incidence of CLABSI. Microbiological profiles and antimicrobial resistance pattern will be reported as pan sensitive, multidrugresistant organism and carbapenem-resistant organism. SQUIRE V.2.0 guidelines will be used for manuscript writing and reporting.

\section{INTRODUCTION}

Healthcare-associated infections (HAI) are a major problem worldwide. ${ }^{1}$ Among HAI, central line-associated bloodstream infections (CLABSI) are the most important cause of morbidity, mortality and prolonged hospital stay, especially in critically ill
What is already known about this subject?

CLABSI is defined as a LCBI (Laboratory confirmed blood stream infection) where central line was in place for greater than two calendar days on the date of the event, with day of device placement being day one, and the line was also in place on the date of the event or the day before.

- In developing countries CLABSI is either un addressed or under reported

- Interventions for reduction of CLABSI when used in form of bundles have proven to be efficacious.

\section{What this study hopes to achieve?}

If successful this can be used at a larger scale for CLABSI prevention both within our institution and other facilities worldwide.

- It might be helpful to understand the spectrum of microorganisms and their antimicrobial resistance.

patients. $^{2}$ Risk of neurodevelopmental impairment significantly increases with one or more episodes of HAI in very low birthweight infants. ${ }^{3}$ Although a $46 \%$ decrease in CLABSI has occurred in hospitals across the USA from 2008 to 2013, an estimated 30100 CLABSI still occur in intensive care units (ICU) and wards of the USA. ${ }^{4}$ Due to many factors in low-resource countries, CLABSI are either unaddressed or under-reported, meaning that the impact of CLABSI-related mortality and morbidity may be much higher than what is reported. In a review of literature, neonatal intensive care unit (NICU) CLABSI rates ranged from 2.6 to 60 cases per 1000 central line days in limited-resource countries in comparison with 2.9 cases per 1000 central line days in the USA. ${ }^{5}$ 
Since Pronovost et al landmark paper in 2006 indicating the usefulness of central line insertion bundles in reducing CLABSI ${ }^{6}$ significant amount of research has led to implementation of line insertion and maintenance bundles in ICU worldwide leading to significant reduction in CLABSI rates. A recent systemic review and meta-analysis showed a risk reduction in CLABSI incidence from 6.4 per 1000 catheter days to 2.5 per 1000 catheter days after implementation of these bundles. Significantly higher risk reduction were also noted in studies having baseline incidence rates of 5 per 1000 catheter days or greater. ${ }^{7}$ A healthcare bundle is defined as a small, straightforward set of evidence-based practices that, when performed collectively and reliably, have been proven to improve patient outcomes. ${ }^{8}$ Merging several evidence-based catheter care practices into one system increases chances of its adherence and eventual success.

Our unit at the Aga Khan University Hospital (AKUH) is a leading tertiary care private sector teaching hospital located in Karachi, Pakistan with a 24-bedded level 3 NICU. We have four bays of five cots each along with four isolation rooms. We admit approximately $800-1000$ patients per year including those born at AKUH and its affiliated hospitals along with those who are transferred from outside hospitals. Our nurse-to-patient ratio is usually $1: 2$ for non-infected patients and 1:1 for culture proven infections. Our hospital has an infection control team which comprises trained infection control nurses and an infectious disease physician. These, along with a dedicated neonatologist, prospectively classify CLABSI according to the US Centers for Disease Control's (CDC) National Health and Safety Network criteria. ${ }^{9}$

Qadir et $a l^{10}$ from our NICU have reported a sustained reduction in multidrug-resistant bloodstream infection (BSI) by using simple evidence-based strategies, which included hand washing certification for all staff, use of chlorhexidine instead of povidone iodine for skin preparation, use of non-sterile gloves for diaper change, implementation of barrier nursing for clinically suspected and culture-proven infections, provision of separate intubation and central line trolley for each room and limiting the use of umbilical catheters to 7 days. These interventions and data are from June 2010 to December 2011. Since then, our utilisation of central lines, especially peripherally inserted central catheters (PICC) has increased due to awareness of its utility but simultaneously our CLABSI rates have climbed high. Our NICU CLABSI rates have been around 9/1000 central line days over the last 5 years (2011-2015), with a line utilisation ratio of 0.36 . Approximately $60 \%$ of our CLABSI are Gram-negative organisms, which are mostly MDR organisms (MDRO) (unpublished data).

Since 2014, we have gradually moved from 12-bedded facility to a 24-bedded unit to accommodate the increasing number of deliveries and referrals. With this increase in bed space, and our high CLABSI rates, it became imperative to initiate a quality improvement programme to reduce CLABSI rates. Therefore, we intend to introduce an evidence-based CLABSI prevention package (CPP) to improve CLABSI rates in our NICU within limited resources.

Objectives: We intend to implement a quality improvement programme by introducing evidence-based CPP and measure its impact on CLABSI rates.

Study design: Preanalysis and postanalysis design using PDCA (Plan DoCheck Act)model.

Settings: AKUH NICU.

Duration:Twelve months (from 1 January2017 to 31 December 2017).

\section{Inclusion criteria}

All patients admitted to AKUH NICU from 1 January 2016 to 31 December 2017, who have central line in place.

\section{OPERATIONAL DEFINITIONS}

1. Central line: a catheter that ends at or close to heart or in any of the great vessels and is to be used for haemodynamic monitoring, infusions and blood sampling. The following will be considered great vessels: aorta, superior vena cava, inferior vena cava, brachiocephalic or innominate veins, internal jugular veins, subclavian veins, external iliac veins and common femoral veins. Central lines that will be included are PICC line, Umbilical arterial and venous lines and central venous line, which includes tunnelled and cuffed or non-tunnelled femoral lines.

2. Laboratory-confirmed bloodstream infection (LCBI): LCBI will be defined as:

a. A patient with a recognised pathogen cultured from one or more blood cultures, where the organism cultured was not related to an infection at another site; or

b. A patient found to have a common skin contaminant (eg, coagulase-negative staphylococci, viridans group streptococci or micrococci, diphtheroids, Bacillus sp, Propionibacterium sp) cultured from two or more blood cultures and one or more of the following signs or symptoms: fever $>38^{\circ} \mathrm{C}$, hypotension or hypothermia $<37^{\circ} \mathrm{C}$, apnoea or bradycardia and where signs, symptoms and positive laboratory results were not related to an infection at another site.

3. CLABSI: according to the CDC, CLABSI is defined as an LCBI where central line or umbilical catheter (UC) was in place for $>2$ calendar days on the date of the event, with day of device placement being on day 1 , and the line was also in place on the date of the event or the day before. For this study, only the first CLABSI will be included for a particular line.

4. Device days: total number of days of exposure to device (central line) by all patients in NICU.

5. CLABSI rates: total number of CLABSI divided by the total number of device days 1000 . 
6. Device utilisation ratio: proportion of patients for which device is used, that is, number of device days/ number of patient days 100 .

7. Pan-sensitive microorganisms: those organisms that are sensitive to all first-line antibiotics.

8. MDRO: multidrug resistance is defined as resistance (R) to at least one agent in three or more tested antimicrobial categories.

9. Carbapenem resistance: it is defined as a result of intermediate (I) or R to imipenem or meropenem.

\section{METHODOLOGY AND DATA COLLECTION}

The study will be performed in three phases.

\section{Phase I: preimplimentation phase}

In this phase, all patients from 1 January 2016 to 31 December 2016, with central line in place for $>2$ calendar days, with the day of line placement as day 1 , will be enrolled.

Patient days, device days, CLABSI rates, line utilisation ratio and hand hygiene compliance over the past 12 months (January-December 2016) will be retrieved from the hospitals infection control data base.

\section{Phase II: implementation phase}

In this phase, evidence-based CPP will be introduced from 1 January 2017 to 31 March 2017. All patients with central line in place for $>2$ calendar days, with day of line placement as day 1 , will be enrolled and followed-up until either transfer from NICU to step-down unit, discharge or death.

\section{Phase III: follow-up phase}

In this phase, all patients from 1 April 2017 to 31 December 2017 , with central line in place for $>2$ calendar days with day of device placement as day 1 , will be enrolled and followed-up until either transfer from NICU to step-down unit, discharge or death.

Prospectively collected data regarding patient days, device days, device utilisation ratio and CLABSI rates will be obtained from hospital infection control surveillance data bases monthly and recorded on a separate excel sheet.

In addition, a team composed of an attending neonatologist, NICU infection control nurse and infection control team would review the patients in the NICU and check the CLABSI cases submitted to ensure data quality. All data will be collected by a NICU fellow not involved in the CPP.

The following data will be recorded for all patients: data regarding gestational age, chronological age, birth weight and current weight, place of birth, length of NICU stay, brief clinical summary along with date, time and reason for removal will be recorded. Dwell time for each line will also be recorded. Total days of parenteral nutrition will also be recorded. Device day at which CLABSI is confirmed will also be noted. Microorganisms will be identified and antibiotic susceptibility profiles will be obtained from laboratory reports and recorded on a predefined proforma. Susceptibility results are usually reported using the category interpretations of susceptible, I, R or not tested. Microorganisms will be classified on the basis of their sensitivity patterns into pan-sensitive, MDRO and carbapenem-resistant organism (CRO).

CPP proforma is shown in online supplementary file.

After a literature search, the following evidence-proven interventions have been identified for reducing CLABSI rates in NICU, which will be implemented over a period of 3 months (Phase II):

1. Hand hygiene

a. Repeated reminders by regular hand hygiene week and champion of the month award ${ }^{11}$ (IA)

b. Non-sterile gloves for routine handling of babies $<1000 \mathrm{~g}^{12}(\mathrm{IA})^{\mathrm{i}}$.

2. Central line insertion

a. Hand hygiene with hospital-approved alcoholbased product or antiseptic-containing soap before and after palpating insertion sites and before and after inserting central line $e^{13-15}$ (IA) ${ }^{\mathrm{i}}$;

b. Use of maximal barrier precautions (including sterile gown, sterile gloves, surgical mask, hat and large sterile drape $)^{1316}(\mathrm{IA})^{\mathrm{i}}$;

c. Disinfect skin with appropriate antiseptic (eg, $2 \%$ chlorhexidine, $70 \%$ alcohol) before catheter insertion $^{131718}(\mathrm{IA})^{\mathrm{i}}$;

d. Use either a sterile transparent semi-permeable dressing or sterile gauze to cover the insertion site $^{19-21}(\mathrm{IA})^{\mathrm{i}}$;

e. Central line insertion certification of physicians $^{22-24}(\mathrm{IB})^{\mathrm{i}}$;

f. Reinforcement of central line kit and implementation of dressing/readjustment $\mathrm{kit}^{25}$ (IA) ${ }^{\mathrm{i}}$;

g. Elective central line insertions at day time.

3. Central line maintenance

a. Closed medication system and two person process for all dressing change and tubing change ${ }^{26}(\mathrm{IB})^{\mathrm{i}}$;

b. Perform hand hygiene with hospital-approved alcohol-based product or antiseptic-containing soap before and after accessing a catheter or changing the dressing ${ }^{13-15}(\mathrm{IA})^{\mathrm{i}}$;

c. Maintain aseptic technique when changing intravenous tubing and when entering the catheter including 'scrub the hub' 202527 (IA) ${ }^{\mathrm{i}}$;

${ }^{\mathrm{i}}$ Ranking system for evidence according to the CDC/Healthcare Infection Control Practice Advisory Committee System: category IA: strongly recommended for implementation and strongly supported by well-designed experimental, clinical or epidemiological studies. Category IB: strongly recommended for implementation and supported by some experimental, clinical or epidemiological studies and a strong theoretical rational. Category $I C$ : required for implementation, as mandated by federal and/or state regulation or standard. Category II: suggested for implementation and supported by suggestive clinical or epidemiological studies or a theoretical rationale or a consensus by a panel of experts. 
d. Evaluate the catheter insertion site daily for signs of infection and to assess dressing integrity. At a minimum, if the dressing is damp, soiled or loose, change it aseptically and disinfect the skin around the insertion site with an appropriate antiseptic $^{18202728}$ (IB) ${ }^{\mathrm{i}}$;

e. Daily review of catheter necessity with prompt removal when no longer essential ${ }^{1329}$ (IB) ${ }^{\mathrm{i} \text { ii }}$;

f. Minimising the access points ${ }^{30}$ (IA) ${ }^{\mathrm{i} i \mathrm{ii}}$;

g. Heprin in TPN $(0.5 \text { Units } / \mathrm{mL})^{31}(\mathrm{IA})^{\mathrm{i} \text { ii }}$.

4. Organism-specific prevention (fungal infections):

a. Fluconazole prophylaxis for babies $<1000 \mathrm{~g}$ (intravenous fluconazole $6 \mathrm{mg} / \mathrm{kg} /$ dose after every 2 days $)^{32}$ (IA) ${ }^{\mathrm{i}}$;

b. Minimise the use of $\mathrm{H} 2$ receptor blocker and proton pump inhibitor ${ }^{33}$ (II) ${ }^{\mathrm{i}}$;

c. Minimise the use of broad-spectrum antibiotics ${ }^{34}$ (II) ${ }^{\mathrm{i}}$.

5. Nurse empowerment ${ }^{35}$

a. Nurse empowerment will be the cornerstone of the project. A dedicated infection control NICU nurse will be identified and empowered to take charge of the overall implementation of CPP.

b. Her/his responsibilities will be as follows:

i. Collect and record daily patient days, device days and device utilisation ratio;

ii. Collect and record all infections in NICU;

iii. Twice monthly meeting with hospitals infection control team to identify CLABSI from the BSI;

iv. Daily assessments of all central line insertion checklists for completeness and deficiencies;

v. Daily feedback to concerned physician and nurse regarding deficiencies in central line insertion and maintenance;

vi. Ensure hand hygiene is practised and its compliance is $100 \%$;

vii. Ensure all components of evidence-based CPP are practised;

viii. Monthly reporting of data to NICU faculty and quarterly to service line chief.

\section{OUTCOMES, ANALYSIS PLAN, RESULTS AND REPORTING}

Our primary outcome is CLABSI rates following implementation of our QI programme. Secondary outcomes may show a decrease in line utilisation ratio, improved hand hygiene compliances and reduction in fungal infections. We also intend to demonstrate microorganism profiles and their antimicrobial resistance patterns.

Final analysis will be done on SPSS V.22.

For all three phases, descriptive analysis will be performed for all variables. Nominal data like age,

\footnotetext{
${ }^{\text {ii }}$ Points $\mathrm{d}$, e and $\mathrm{f}$ will be reviewed daily by using sticker (attached).
}

weight, gestational age, length of hospital stay, catheter dwell time and TPN days will be presented as mean \pm SD . Categorical data like source of admission, outcomes, birth weight and gestational age categories, diagnosis, catheter type will be presented as frequencies and percentages.

To compare the effect of intervention, we will use independent sample t-test for continuous outcomes, whereas $\mathrm{X}^{2}$ test will be used for categorical outcomes. Relative risk ratios, $95 \%$ CIs and $p$ values will be determined for all outcomes. Probability values $<0.05$ using two-sided tests will be considered significant. Incidence density will be calculated and Poisson regression will be used to determine factors associated with incidence of CLABSI. Microbiological profiles and their antimicrobial resistance pattern will be reported as pan sensitive, MDRO and CRO. We will use SQUIRE V.2.0. Guidelines for reporting our findings. ${ }^{36}$

\section{DISCUSSION AND CONCLUSION}

CPP is designed to improve the quality of care by reducing CLABSI in our unit. The project will help in identifying and comparing the risk factors for development of CLABSI and the impact of evidence-based interventions in reducing it. It will also help in understanding the spectrum of microorganisms related to the development of CLABSI and their antimicrobial resistance patterns.

We feel confident that we will be able to achieve this by meticulous implementation and monitoring of this programme. This research will shed light on the extent of this unaddressed and under-reported problem, especially in our part of the world and will possibly find cost-effective solutions which will lead to long-term changes in our NICU protocols and may be helpful for other similar units in low-income countries.

Contributors ASsH and SRA conceptualised and wrote the initial draft. SA reviewed and revised the manuscript. ASsH, SA,SD and JZ contributed to the development and implementation of the study interventions. AR contributed to the study design and analysis plan. All authors contributed equally in the final approval of the submitted version of the manuscript.

Competing interests None declared.

Ethics approval Obtained.

Provenance and peer review Not commissioned; externally peer reviewed.

Open Access This is an Open Access article distributed in accordance with the Creative Commons Attribution Non Commercial (CC BY-NC 4.0) license, which permits others to distribute, remix, adapt, build upon this work non-commercially, and license their derivative works on different terms, provided the original work is properly cited and the use is non-commercial. See: http://creativecommons.org/ licenses/by-nc/4.0/

(c) Article author(s) (or their employer(s) unless otherwise stated in the text of the article) 2017. All rights reserved. No commercial use is permitted unless otherwise expressly granted.

\section{REFERENCES}

1. Dudeck MA, Edwards JR, Allen-Bridson K, et al. National healthcare safety network report, data summary for, 2013. 
2. Siempos II, Kopterides P, Tsangaris I, et al. Impact of catheterrelated bloodstream infections on the mortality of critically ill patients: a meta-analysis. Crit Care Med 2009;37:2283-9.

3. Stoll BJ, Hansen NI, Adams-Chapman I, et al. Neurodevelopmental and growth impairment among extremely low-birth-weight infants with neonatal infection. JAMA 2004;292:2357-65.

4. CDC national and state healthcare-associated infections progress report. 2014 http://www.cdc.gov/HAl/pdfs/progress-report/haiprogress-report.pdf

5. Rosenthal VD. Central line-associated bloodstream infections in limited-resource countries: a review of the literature. Clin Infect Dis 2009;49:1899-907.

6. Pronovost P, Needham D, Berenholtz S, et al. An intervention to decrease catheter-related bloodstream infections in the ICU. N Engl J Med 2006;355:2725-32.

7. Ista $\mathrm{E}$, van der Hoven B, Kornelisse RF, et al. Effectiveness of insertion and maintenance bundles to prevent central-lineassociated bloodstream infections in critically ill patients of all ages: a systematic review and meta-analysis. Lancet Infect Dis 2016;16:724-34.

8. Resar R, Pronovost P, Haraden C, et al. Using a bundle approach to improve ventilator care processes and reduce ventilator-associated pneumonia. Jt Comm J Qual Patient Saf 2005;31:243-8.

9. O'Grady NP, Alexander M, Burns LA, et al. "Guidelines for the Prevention of Intravascular Catheter-related Infections". Clinical Infectious Diseases 52 2011:1087-99.

10. Qadir M, et al. "Effectiveness of simple strategies in reducing multidrug resistant blood stream infections in neonatal intensive care unit of tertiary care hospital in Karachi, Pakistan". JPMA. The Journal of the Pakistan Medical Association 65 2015;1:72-5.

11. Pittet D, Hugonnet $S$, Harbarth $S$, et al. Effectiveness of a hospitalwide programme to improve compliance with hand hygiene. The Lancet 2000;356:1307-12.

12. Kaufman DA, Blackman A, Conaway MR, et al. Nonsterile glove use in addition to hand hygiene to prevent late-onset infection in preterm infants: randomized clinical trial. JAMA Pediatr 2014;168:909-16.

13. Pronovost P. Interventions to decrease catheter-related bloodstream infections in the ICU: the Keystone Intensive Care Unit Project. Am J Infect Control 2008;36:S171.e1-S171.e5.

14. CDC. Guideline for hand hygiene in health-care settings: recommendations of the Healthcare infection control practices advisory committee and the HICPAC/SHEA APIC/IDSA hand hygiene task force. MMWR 2002;51:PR-1-45.

15. Boyce JM, Pittet D. Healthcare Infection Control Practices Advisory CommitteeHICPAC/SHEA/APIC/IDSA Hand Hygiene Task Force. Guideline for hand hygiene in health-care settings. Recommendations of the healthcare infection control practices advisory committee and the HIPAC/SHEA/APIC/IDSA hand hygiene task force. Am J Infect Control 2002;30:S1-46.

16. Raad II, Hohn DC, Gilbreath BJ, et al. Prevention of central venous catheter-related infections by using maximal sterile barrier precautions during insertion. Infect Control Hosp Epidemiol 1994;15:231-8.

17. Chaiyakunapruk N, Veenstra DL, Lipsky BA, et al. Chlorhexidine compared with povidone-iodine solution for vascular catheter-site care: a meta-analysis. Ann Intern Med 2002;136:792-801.

18. Mimoz O, Villeminey S, Ragot S, et al. Chlorhexidine-based antiseptic solution vs alcohol-based povidone-iodine for central venous catheter care. Arch Intern Med 2007;167:2066-72.
19. McGee DC, Gould MK. Preventing complications of central venous catheterization. N Engl J Med 2003;348:1123-33.

20. Maki DG, Stolz SS, Wheeler S, et al. A prospective, randomized trial of gauze and two polyurethane dressings for site care of pulmonary artery catheters: implications for catheter management. Crit Care Med 1994;22:1729-37.

21. Gillies D, O'Riordan L, Carr D, et al. Gauze and tape and transparent polyurethane dressings for central venous catheters. Cochrane Database Syst Rev 2003;4:CD003827.

22. Kyle KS, Myers JS. Peripherally inserted central catheters. Development of a hospital-based program. $J$ Intraven Nurs 1990;13:287-90.

23. Linck DA, Donze A, Hamvas A. Neonatal peripherally inserted central catheter team. Evolution and outcomes of a bedside-nurse-designed program. Adv Neonatal Care 2007;7:22-9.

24. Soifer NE, Borzak S, Edlin BR, et al. Prevention of peripheral venous catheter complications with an intravenous therapy team: a randomized controlled trial. Arch Intern Med 1998;158:473-7.

25. Garland JS, Alex CP, Sevallius JM, et al. Cohort study of the pathogenesis and molecular epidemiology of catheter-related bloodstream infection in neonates with peripherally inserted central venous catheters. Infect Control Hosp Epidemiol 2008;29:243-9.

26. Aly $\mathrm{H}$, Herson $\mathrm{V}$, Duncan $\mathrm{A}$, et al. Is bloodstream infection preventable among premature infants? Atale of two cities Pediatrics 2005;115:1513-8.

27. Salzman MB, Isenberg HD, Shapiro JF, et al. A prospective study of the catheter hub as the portal of entry for microorganisms causing catheter-related sepsis in neonates. J Infect Dis 1993;167:487-90.

28. Crnich CJ, Maki DG. The promise of novel technology for the prevention of intravascular device-related bloodstream infection. II. Long-term devices. Clin Infect Dis 2002;34:1362-8.

29. Berenholtz SM, Pronovost PJ, Lipsett PA, et al. Eliminating catheterrelated bloodstream infections in the intensive care unit. Crit Care Med 2004;32:2014-20.

30. McCarthy MC, Shives JK, Robison RJ, et al. Prospective evaluation of single and triple lumen catheters in total parenteral nutrition. JPEN $J$ Parenter Enteral Nutr 1987;11:259-62.

31. Birch P, Ogden S, Hewson M. A randomised, controlled trial of heparin in total parenteral nutrition to prevent sepsis associated with neonatal long lines: the Heparin in Long Line Total Parenteral Nutrition (HILLTOP) trial. Arch Dis Child Fetal Neonatal Ed 2010;95:F252-F257.

32. Cleminson J, Austin N, McGuire W. "Prophylactic systemic antifungal agents to prevent mortality and morbidity in very low birth weight infants". The Cochrane Library 2015.

33. Saiman L, Ludington $\mathrm{E}$, Pfaller $\mathrm{M}$, et al. Risk factors for candidemia in neonatal intensive care unit patients. Pediatr Infect Dis $J$ 2000;19:319-24

34. Noyola DE, Fernandez M, Moylett EH, et al. Ophthalmologic, visceral, and cardiac involvement in neonates with candidemia. Clin Infect Dis 2001;32:1018-23.

35. Ceballos K, Waterman K, Hulett $\mathrm{T}$, et al. Nurse-driven quality improvement interventions to reduce hospital-acquired infection in the NICU. Adv Neonatal Care 2013;13:154-63.

36. Goodman D, Ogrinc G, Davies L, et al. Explanation and elaboration of the SQUIRE (Standards for Quality Improvement Reporting Excellence) Guidelines, V.2.0: examples of SQUIRE elements in the healthcare improvement literature. BMJ Qual Saf 2016;25:e7. 